\title{
Clinical Assessment of Macular Involvement and Its Correlation to the Severity of Diabetic Retinopathy in Uncomplicated Type 2 Diabetes Mellitus Patients
}

\author{
Kandasamy Kalaivani ${ }^{1}$, Purnananda Mishra² ${ }^{2}$ Mishra Amrith ${ }^{3}$ \\ ${ }^{1}$ Department of Ophthalmology, Rajah Muthiah Medical College, Annamalai University, Chidambaram, Tamilnadu, \\ India. ${ }^{2}$ Department of Ophthalmology, Rajah Muthiah Medical College, Annamalai University, Chidambaram, \\ Tamilnadu, India. ${ }^{3}$ Department of Community Medicine, Mahatma Gandhi Medical College, Puducherry, India.
}

\section{ABSTRACT}

\section{BACKGROUND}

India has roughly $50 \%$ of the world's burden of diabetes and is expected to double by the year 2025. Macular oedema is the leading cause of central vision loss among the diabetic population. Our aim was to detect the presence of macular oedema in type 2 diabetic patients and correlate it to the type and severity of diabetic retinopathy.

\section{METHODS}

This was a cross-sectional study done on two hundred patients with type 2 diabetes mellitus fulfilling our inclusion criteria. After entering patients' details, complete history including duration and treatment of diabetes, detailed clinical slit lamp examination, with biomicroscopy/IDO was done and the fundus findings were documented using the fundus camera, and retinopathy was graded according to the ETRS Classification system. Patients' glucose level, HbA1c, cholesterol, urea and creatinine values were collected. BMI was calculated from their height and weight. Macular findings were documented and correlated to the patient variables and also to the severity of diabetic retinopathy and analysed using the Chi-square test.

\section{RESULTS}

Out of 200 patients, 128 were male and 72 were female. Our incidence of CSME was $14 \%$ and was more prevalent in patients with Non-Proliferative Diabetic Retinopathy and they all showed statistically significant reduction in their visual acuity. BMI, cholesterol, duration of diabetes, HbA1c and insulin therapy didn't show any significant correlation to the occurrence of CSME.

\section{CONCLUSIONS}

Periodic fundus evaluation to detect macular oedema associated with retinopathy is mandatory for diabetic patients. Proper diagnosis and early intervention will help in reducing central vision loss in diabetic patients.

\section{KEY WORDS}

Type 2 Diabetes Mellitus, Diabetic Macular Oedema, Diabetic Retinopathy
Corresponding Author: Dr. Kandasamy Kalaivani, Professor,

Department of Ophthalmology, Vinayaka Mission's Medical College, Karaikal, Puducherry, India. E-mail:drkvani@gmail.com

DOI: $10.14260 /$ jemds/2020/339

Financial or Other Competing Interests: None.

How to Cite This Article:

Kalaivani K, Mishra P, Amrith M. Clinical assessment of macular involvement and its correlation to the severity of diabetic retinopathy in uncomplicated type 2 diabetes mellitus patients. J. Evolution Med. Dent. Sci. 2020;9(19):1554-1558, DOI: $10.14260 /$ jemds $/ 2020 / 339$

Submission 13-03-2020,

Peer Review 24-04-2020,

Acceptance 30-04-2020,

Published 11-05-2020.

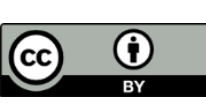




\section{BACKGROUND}

Diabetes Mellitus is the leading cause of morbidity in India. The overall prevalence of diabetes in India is $7.3 \%$. India has roughly $50 \%$ of the world's burden of diabetes and is expected to double by the year 2025. Diabetic retinopathy is the most common micro vascular complication and macular oedema is the leading cause of blindness among the diabetic population. Macular oedema is more prevalent in type 2 than type 1 diabetes mellitus. This study was done to detect the presence of macular oedema in uncomplicated type 2 diabetic patients and to correlate the same to the severity of retinopathy.

\section{Pathogenesis of Diabetic Retinopathy}

Diabetic retinopathy can be divided into non-proliferative and proliferative stages. The main damage occurs in the pericytic cells of the retinal capillaries producing micro aneurysm and leak with the formation of haemorrhage and exudates in the surrounding retina. With further progression of the disease, progressive ischemia of the retina occurs because of capillary non-perfusion. The hypoxic retina liberates angiogenic substances which lead on to the formation of new vessels on or near the optic disc (NVD) or elsewhere in the retina (NVE). Neovascularisation is the hallmark of proliferative diabetic retinopathy. These new vessels are fragile and prone for bleeding resulting in retinal and vitreous haemorrhage. Long standing vitreous haemorrhage causes fibrosis, traction bands, retinal tears and tractional retinal detachment. It may also cause neovascular glaucoma. Type 2 diabetic patients with diabetic age less than 5 years have $24 \%$ chance of developing diabetic retinopathy and $2 \%$ chance for proliferative diabetic retinopathy and the incidence increases with the longer duration of diabetes mellitus.

\section{Pathophysiology of Diabetic Macular Oedema}

With hyperglycaemia there is disorganization of inter endothelial junctions causing leakage of the fluid through the capillary walls into the retinal tissue. In the foveal region, outer plexiform layer can host cystoid spaces of very large proportions. Most cases of clinically apparent oedema are in this region only. Presence of hard exudates is one of the most characteristic clinical finding in diabetic macular oedema. Accumulation of hard exudates in the foveal centre lead to the loss of central vision.

\section{Risk Factors for Diabetic Macular Oedema}

Hyperglycaemia is an identified risk factor for the progression of retinopathy as well as diabetic macular oedema. Older onset patients requiring insulin have a higher incidence of macular oedema likely to be due to poor glycaemic control. Duration of diabetes is strongly associated with prevalence of macular oedema, retinopathy progression and other diabetic complications. Few proportions of type 2 diabetic patients may present with poor vision due to macular oedema even in their first visit. Dyslipidemia has been implicated as an independent risk factor for DME. 1,2
Hypertension significantly exacerbates the vision loss due to macular oedema. ${ }^{3}$

\section{Clinical Presentation of DME}

Macular oedema is the most frequent cause of visual impairment in patients with diabetic retinopathy. Macular oedema is manifested as retinal thickening primarily due to exudation from the incompetent macular capillaries. The ETDRS investigators classified macular oedema by its severity. It was defined as clinically significant macular oedema (CSME) if any of the following features were present (1) thickening of the retina at or within $500 \mu \mathrm{m}$ of the centre of the macula, (2) hard exudates at or within $500 \mu \mathrm{m}$ of the centre of the macula if associated with thickening of the adjacent retina, or (3) a zone of retinal thickening of one disc area or larger, any part of which is within one disc diameter from the centre of the macula.

\section{METHODS}

Our study was a cross-sectional study conducted on type 2 diabetic patients uncomplicated with hypertension, cardiac or renal involvement who attended our outpatient department from November 2018-2019. Based on our local population and on the prevalence of $9 \%$ type 2 diabetes, with $95 \%$ confidence level and $5 \%$ absolute precision, our sample size was estimated to be $126^{4}$ and we collected 200 samples. Institutional Ethical committee approval obtained. The diagnosis of diabetes was made according to WHO criteria reported by the WHO study group in $1985^{5}$. Two hundred proven cases of Type 2 diabetic patients of both sexes, more than 40 years of age belonging to all socio-economic state were included in the study. Informed consent was taken. Patients with hypertension, chronic kidney disease and congestive cardiac failure and those who underwent laser treatment for retina were excluded from the study. Patient details with their height and weight measurement, duration and treatment of diabetes were entered. Body mass index was calculated for all patients from their height and weight data using the formula $\mathrm{BMI}=$ weight in $\mathrm{Kg}$ / height in metres squared. Patients' blood sugar, HbA1c and cholesterol values were recorded. Detailed clinical examination like slit-lamp bio microscopy/Indirect ophthalmoscopy was done and the fundus findings were documented using the fundus camera and the retinopathy was graded according to the ETDRS Classification system ${ }^{6}$. Macular findings were documented and correlated to the duration of diabetes, HbA1c values and also to the severity of diabetic retinopathy and analysed using the chi-square test.

\section{RESULTS}

Out of the 200 study population, $128(64 \%)$ were male and 72 (36\%) were female. Mean age of the patients was 55 (mean \pm SD was $55.04 \pm 10.499$. The mean BMI was 23.5 for women and 22 for men and it didn't show any significant correlation to the occurrence of CSME ( $t$ test, P-value 0.992). 


\begin{tabular}{|c|c|c|c|c|c|}
\hline Variable & CSME & $\mathbf{N}$ & Mean \pm SD & t Value & P Value \\
\hline \multirow{2}{*}{ Age in years } & With CSME & 28 & $54.25 \pm 8.79$ & \multirow{2}{*}{-0.429} & \multirow{2}{*}{0.67} \\
\hline & Without CSME & 172 & $55.04 \pm 10.49$ & & \\
\hline \multirow{2}{*}{ Diabetic Age } & With CSME & 28 & $8.10 \pm 4.31$ & \multirow{2}{*}{-0.446} & \multirow{2}{*}{0.657} \\
\hline & Without CSME & 172 & $8.53 \pm 6.44$ & & \\
\hline \multirow{2}{*}{ BMI } & With CSME & 28 & $22.67 \pm 3.12$ & \multirow{2}{*}{0.011} & \multirow{2}{*}{0.992} \\
\hline & Without CSME & 172 & $22.66 \pm 3.44$ & & \\
\hline \multirow{2}{*}{ FBS } & With CSME & 28 & $181.32 \pm 55.31$ & \multirow{2}{*}{2.41} & \multirow{2}{*}{0.02} \\
\hline & Without CSME & 172 & $153.27 \pm 67.23$ & & \\
\hline \multirow{2}{*}{ PPBS } & With CSME & 28 & $262.99 \pm 86.91$ & \multirow{2}{*}{1.905} & \multirow{2}{*}{0.065} \\
\hline & Without CSME & 172 & $229.21 \pm 87.89$ & & \\
\hline \multirow{2}{*}{ HBA1C } & With CSME & 28 & $7.60 \pm 1.62$ & \multirow{2}{*}{1.37} & \multirow{2}{*}{0.179} \\
\hline & Without CSME & 171 & $7.41 \pm 1.63$ & & \\
\hline \multirow{2}{*}{ Sr Choles } & With CSME & 28 & $158.00 \pm 28.97$ & \multirow{2}{*}{-0.654} & \multirow{2}{*}{0.517} \\
\hline & Without CSME & 172 & $161.98 \pm 35.03$ & & \\
\hline \multirow{2}{*}{ Urea } & With CSME & 28 & $31.34 \pm 8.18$ & \multirow{2}{*}{1.468} & \multirow{2}{*}{0.151} \\
\hline & Without CSME & 172 & $28.93 \pm 7.12$ & & \\
\hline \multirow{2}{*}{ Creatinine } & With CSME & 28 & $0.87 \pm 0.18$ & \multirow{2}{*}{-0.222} & \multirow{2}{*}{0.826} \\
\hline & Without CSME & 172 & $0.88 \pm 0.21$ & & \\
\hline \multicolumn{6}{|c|}{ Table 1. Patient Variables } \\
\hline
\end{tabular}
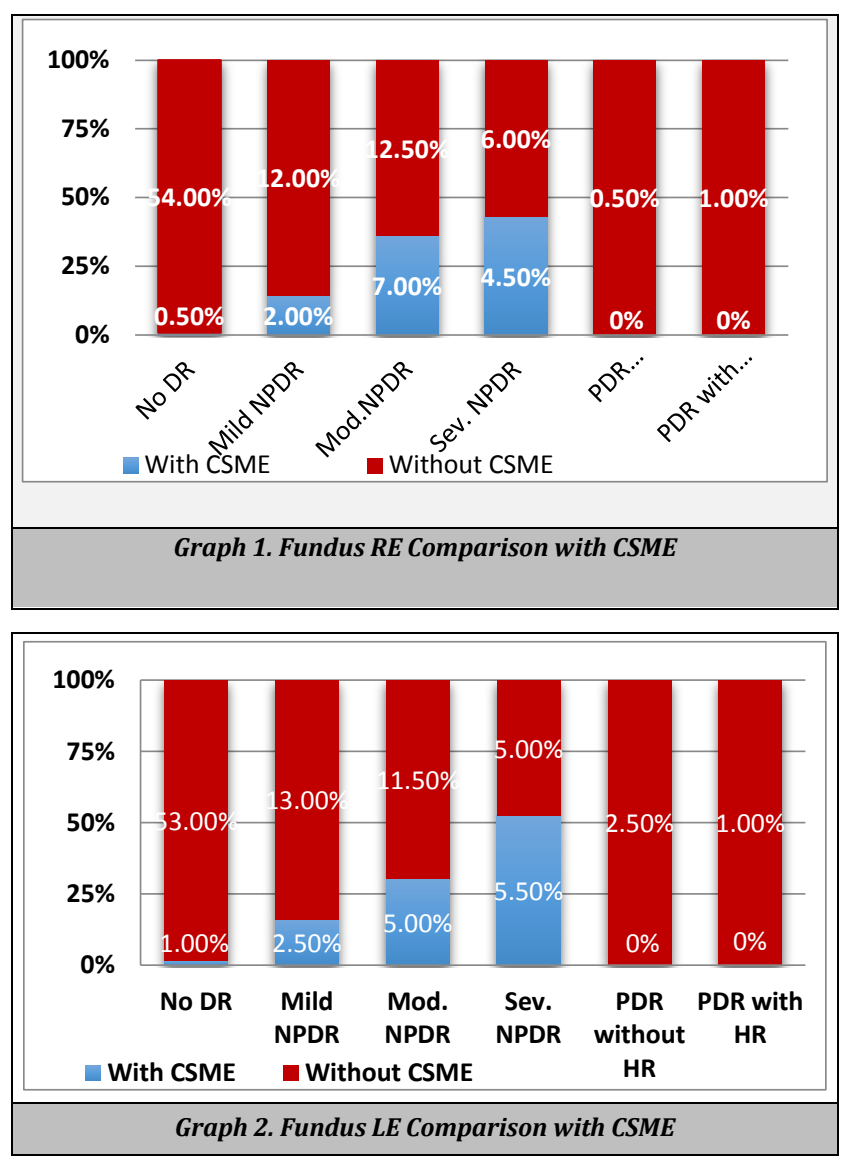

There is no significant difference in the mean cholesterol value between the CSME and non-CSME group and hypercholesterolemia didn't influence the occurrence of macular oedema ( $t$ test, p-value 0.517). Since patients with chronic kidney disease and hypertension were excluded from the study, urea and creatinine values were also not positively correlated to macular oedema.

The mean diabetic age of the CSME group was 8.1 and the non-CSME group was 8.5 which is not significant statistically (ref table 1). Our sample included newly diagnosed diabetic cases to a maximum of 30 years of diabetes. There were 57 patients $(28.5 \%)$ with diabetic age of less than 5 years and another 57 patients (28.5\%) were between 5-10 years of diabetic duration; $39.5 \%$ had duration between $10-20$ years and $3.5 \%$ had a longer diabetic duration of more than 20 years. Among the total 200 patients, 91 (45.5\%) had diabetic retinopathy- 28 (14\%) had mild non-proliferative diabetic retinopathy (NPDR), 39 (19.5\%) had moderate NPDR, 21(10.5\%) had severe NPDR, $0.5 \%$ had proliferative retinopathy (PDR) without high risk and 1\% had PDR with high risk. 28 patients (14\%) had CSME. This includes 17 male and 11 female patients (Fisher's Exact Test - * p value 0.678).

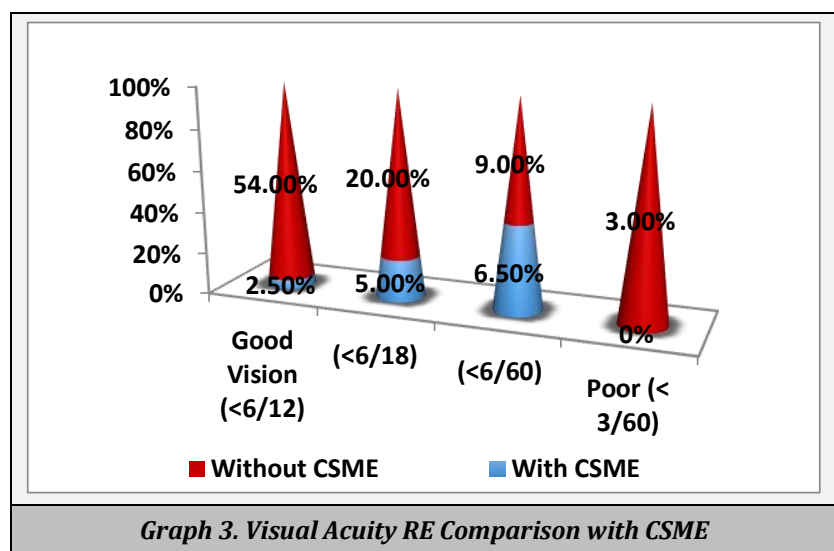

Graph 3. Visual Acuity RE Comparison with CSME

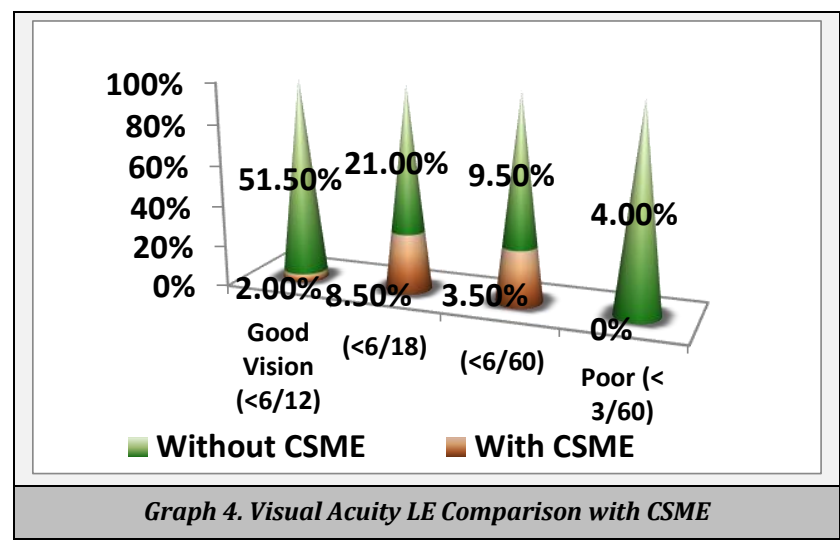

In both CSME and non CSME groups, majority of patients ( $>60 \%$ ) were on insulin therapy and the remaining were on oral hypoglycaemic agents. Though patients who were on insulin had higher incidence of CSME than those on oral hypoglycaemic drugs, but it was not statistically significant (Chi Square Test- p-value 0.72) 75\% of the total sample population had good diabetic control with $\mathrm{HbA} 1 \mathrm{c}$ value of 6.5-7.5; $10 \%$ had fair control with 7.5-8.5 and 15\% had poor control with HbA1c level above 8.5. CSME group had the mean HbA1c value of 7.6 while others had the mean of 7.4 and there was no positive correlation of HbA1c with CSME $(t$ test, $p$-value 0.179). CSME was found to be more prevalent in patients with Non-Proliferative Diabetic Retinopathy and there was increased prevalence of CSME with increased severity of DR (Chi Square Test p-value 0.0001). The majority of the patients with CSME showed a significant reduction in their visual acuity of $<6 / 60$ (Chi Square Test P-value 0.0001).

\section{DISCUSSION}

Unlike others, our study highlights the original impact of diabetes on macula uninfluenced by other variables since renal, cardiac and hypertensive patients were excluded. Body Mass Index didn't show any significant correlation to the occurrence of CSME in our study which was similar to the report of the meta-analysis of 27 articles done by Yue Zhou et 
al who evaluated the association of BMI with DR and proved that neither being overweight nor obesity conferred an increased risk of $\mathrm{DR}^{7}$ while H. I. Salti et al in their study showed that elevated BMI was associated with increased prevalence of macular oedema in type 2 diabtetes. ${ }^{8}$

Hypercholesterolemia was not associated with the increased incidence of CSME in our study which resembles the previous study done by Benarous $\mathrm{R}$ et al who also found that serum lipid levels were not related to DR or $\mathrm{DME}^{9}$ whereas ETDRS and DCCT studies showed a positive correlation between increased lipid levels and development of DME; Singh Luxmi et al also proved in their study that DME was associated with elevated fasting and postprandial blood sugar and hyper triglyceridemia. ${ }^{10}$

Panagiotoglou TD et al studied the effect of Atorvastatin in type 2 diabetic patients with elevated serum cholesterol levels and found that fluorescein leakage and severity of hard exudates were reduced with the use of Atorvastatin. ${ }^{11} \mathrm{~A}$ study by Gupta et al from North India concluded that oral atorvastatin caused a reduction in the hard exudates in CSME patients and hence it could be an adjuvant therapy in the management of DME. Dyslipidemia had been proved to be an independent risk factor for DME by Ferris FL et al. ${ }^{12}$ Longer duration of diabetes in our study didn't show a positive correlation on the prevalence of CSME but Ong Ming Jew et al study showed that the duration of diabetes was significantly higher in CSME group. ${ }^{13}$ Klein R, Moss SE et al study also showed that increased duration of diabetes was strongly associated with prevalence of macular oedema, retinopathy progression and other diabetic complications ${ }^{14,3}$ No statistically significant correlation was found between $\mathrm{HbA1C}$ and CSME in our study whereas both WESDR and DCCT studies showed a positive impact of $\mathrm{HbA1C}$ on the occurrence and progression of DME. CURES study also documented that every $2 \%$ increase in $\mathrm{HbA} 1 \mathrm{C}$ level was associated with a 1.7 fold increase in the risk of DME. This might be due to the fact that $75 \%$ of our study population had a good control of diabetes with $\mathrm{HbA1C}$ value less than 7.5.

Among the total 200 patients in our study, $45.5 \%$ had diabetic retinopathy out of which $14 \%$ presented with mild non-proliferative diabetic retinopathy (NPDR), 19.5\% moderate NPDR, $10.5 \%$ severe NPDR, $0.5 \%$ proliferative retinopathy (PDR) without the high risk and 1\% PDR with high risk. Prevalence of CSME was $14 \%$ in our study whereas Lubna Ahmad etal ${ }^{15}$ study showed that the overall prevalence of DME with DR was 29\% and DME was more in patients with NPDR. ${ }^{13}$ United Kingdom Prospective Study (UKPDS) showed 39\% DR prevalence. Andhra Pradesh Eye Disease Study (APDES) showed 22.4\% DR prevalence. Chennai Urban Rural Epidemiological Study (CURES) showed retinopathy prevalence rate of $17.65^{16}$ and in Sankara Nethralaya (SNDREAMS) study ${ }^{17}$ prevalence rate was $18 \%$ and it showed more incidence of CSME in patients with moderate NPDR group.

\section{CONCLUSIONS}

Periodic fundus evaluation to detect macular oedema associated with retinopathy is mandatory for diabetic patients. Proper diagnosis and early intervention will help in reducing the central vision loss in these patients.

\section{Limitations}

Our diagnosis of Diabetic Macular Oedema was based on slit lamp biomicroscopic findings. Hence clinically non-significant DME with no exudates and without significant reduction in visual acuity remained undetected thereby causing underestimation of DME.

\section{ACKNOWLEDGEMENT}

Authors acknowledge Dr. Felix, Professor, Department of Community Medicine, Rajah Muthiah Medical College, Chidambaram and Dr. Sathesh, Statistician cum Lecturer, Department of Community Medicine, Vinayaka Missions' Medical College, Karaikal for their help in statistical analysis.

\section{REFERENCES}

[1] Zander E, Herfurth S, Bohi B, et al. Maculopathy in patients with diabetes type 1 and type 2: associations with risk factors. Br J Ophthalmolo 2000;84(8):871-6.

[2] Xie J, Ikram MK, Cotch MF, et al. Association of diabetic macular oedema and proliferative diabetic retinopathy with cardiovascular disease: a systematic review and meta-analysis. JAMS Ophthalmol 2017;135(6):586-93.

[3] Moss SE, Klein R, Klein BE. The 14 year incidence of visual loss in a diabetic population. Ophthalmology 1998;105(6):998-1003.

[4] Manaviat MR, Afkami R, Shoja MR. Retinopathy and microalbuminuria in type II diabetic patients. BMC Ophthalmol 2004;4:9.

[5] WHO Study Group on Diabetes Mellitus \& World Health Organization (1985). Diabetes Mellitus: report of a WHO study group (meeting held in Geneva from 11-16 February 1985). World Health Organization.

[6] Goldberg MF, Jampol LM. Knowledge of diabetic retinopathy before and 18 years after the Airlie house symposium on the treatment of diabetic retinopathy. Ophthalmology 1987;94(7):741-6.

[7] Zhou Y, Zhang Y, Ke S, et al. Body mass index and risk of diabetic retinopathy: a meta-analysis and systematic review. Medicine (Baltimore) 2017;96(22):e6754.

[8] Salti HI, El Haibi C, Nasrallah MP, et al. Elevated body mass index is associated with higher prevalence of macular oedema in patients with type 2 diabetes. Investigative Ophthalmology \& Visual Science 2006;47:338.

[9] Benarous R, Sasongko MB, Qureshi S, et al. Differential Association of serum lipids with diabetic retinopathy and diabetic macular oedema. Invest Ophthalmol Vis Sci 2011;52(10):7464-9.

[10] Luxmi S, Ritika M, Lubna M, et al. Diabetic macular oedema and its association to risk factors in an urban north Indian population. J Clinical Ophthalmology 2018;2(2):86-91.

[11] Panagiotoglou TD, Ganotakis ES, Kymionis GD, et al. Atorvastatin for diabetic macular oedema in patients with diabetes mellitus and elevated serum cholesterol. Ophthalmic Surg Lasers Imaging 2010;41(3):316-22. 
[12] Ferris FL 3rd, Chew EY, Hoogwerf BJ. Serum lipids and diabetic retinopathy. Early Treatment Diabetic Retinopathy Study Research Group. Diabetes Care 1996;19(11):1291-3.

[13] Jew OM, Peyman M, Chen TC, et al. Risk factors for clinically significant macular oedema in a multiethnics population with type 2 diabetes. Int J Ophthalmol 2012;5(4):499-504.

[14] Klein R, Klein BE, Moss SE, et al. The Wisconsin epidemiologic study of diabetic retinopathy. XV. The long-term incidence of macular oedema. Ophthalmology 1995;102(1):7-16.
[15] Ahmad L, Khan TH, Bundela RK, et al. Prevalence of diabetic macular oedema in association with severity of diabetic retinopathy. J Med Sci \& Clin Res 2017;5(2):17847-52.

[16] Rema M, Premkumar S, Anitha B, et al. Prevalence of diabetic retinopathy in urban India: The Chennai Urban Rural Epidemiological Study (CURES) eye study, I. Invest Ophthalmol Vis Sci 2005;46(7):2328-33.

[17] Raman R, Rani PK, Rachepalle SR, et al. Prevalence of diabetic retinopathy in India: Sankara Nethralaya Diabetic Retinopathy Epidemiology and Molecular Genetics Study report 2. Ophthalmology 2009;116(2):311-8. 